\title{
Caos, complexidade e Lingüística Aplicada: diálogos transdisciplinares
}

Antônio Carlos Soares Martins

Universidade Estadual de Montes Claros

Júnia de Carvalho Fidelis Braga

Universidade Federal de Minas Gerais

RESUMO: Neste texto, apresentamos um panorama de estudos na área da Lingüística Aplicada que discutem questões relativas ao processo de desenvolvimento de segunda língua, bem como aspectos relacionados aos ambientes interacionais de aprendizagem na perspectiva das teorias do Caos e Complexidade. Para isso, discutimos as principais noções da perspectiva da complexidade, retomando alguns aspectos da física clássica e contemporânea.

PALAVRAS CHAVE: teorias do caos e da complexidade; lingüística aplicada; transdisciplinaridade; aprendizagem de línguas.

ABSTRACT: In this paper, we present a panorama of studies in the area of Applied Linguistics that discuss questions regarding second language development as well as aspects related to interactional learning environments in the light of Chaos and Complexity theories. To accomplish this, we discuss the main notions of complexity through the rethinking of some aspects of classic and contemporary physics.

KEY-WORDS: chaos and complexity theories; applied linguistics; transdisciplinarity; language learning.

Surgida inicialmente nas ciências naturais, a teoria da complexidade tem sido cada vez mais utilizada para a compreensão de sistemas humanos e sociais. Como destaca Morin (1990), a vida humana é um fenômeno de auto-eco-organização extraordinariamente complexo e, portanto, os fenômenos antropossociais não podem obedecer a princípios de inteligibilidade menos complexos do que aqueles requeridos para os fenômenos naturais.

Nesse sentido, apresentamos contribuições na área da Lingüística Aplicada que compartilham o pensamento de que existem similaridades entre as proposições das teorias do caos e da complexidade e os fenômenos 
que se manifestam no processo de desenvolvimento ${ }^{1}$ de uma segunda língua. ${ }^{2}$

Compartilhamos a idéia de Demo (2002) de que o conhecimento e a aprendizagem são considerados atividades imbuídas de processos não lineares, tanto em seu processo de formação e reconstrução quanto em sua tessitura interna, uma vez que sinalizam fenômenos tipicamente complexos, porque não se exaurem em alinhamentos lógicos.

\section{O que é a complexidade?}

À primeira vista, a complexidade é um fenômeno que acolhe uma extrema quantidade de interações e de interferência entre um grande número de unidades. Porém, a complexidade não compreende apenas quantidades de unidades e interações que desafiam até mesmo as possibilidades de cálculo; "a complexidade compreende, efetivamente, o tecido de acontecimentos, ações, interações, retroações, determinações, acasos que constituem o nosso mundo fenomenal" (MORIN, 1990, p. 20).

Na ciência, a complexidade ainda é um campo novo, abrangente, sem uma definição exata e limites palpáveis, o que se dá, principalmente, devido ao fato de a pesquisa nessa área tentar explicar questões que desafiam todas as categorias convencionais (WALDROP, 1992). Algumas dessas questões, levantadas por Waldrop (1992), estão aqui resumidas em:

\footnotetext{
${ }^{1}$ Embora o termo aquisição seja consagrado nos estudos em Lingüística Aplicada, preferimos, neste trabalho, utilizar os termos 'aprendizagem', 'desenvolvimento' e 'emergência', que nos parecem mais adequados a uma perspectiva teórica que considera toda a complexidade desses processos. Mantemos o termo 'aquisição' ao referirmo-nos a outros trabalhos que utilizam esse termo.

2 Neste trabalho, utilizamos o termo 'segunda língua' para referirmo-nos a qualquer outra língua aprendida subseqüentemente à língua materna. Reconhecemos as distinções e dualidades existentes na literatura da área, mas alinhamo-nos a uma perspectiva teórica que utiliza o termo 'segunda língua' para referir-se, de forma geral, ao desenvolvimento de outras línguas além da língua materna (ELLIS, 2003). Utilizamos o termo 'língua estrangeira' apenas em momentos particulares, quando for relevante marcar que se trata de aprendizagem por meio de instrução formal em sala de aula em contexto em que a língua em desenvolvimento não é falada como língua materna.
} 
- Por que a hegemonia política da União Soviética entra em colapso em 1989? Por que o colapso do comunismo foi tão completo e tão rápido?

- Por que o mercado de ações caiu mais de 500 pontos em um único dia em outubro de 1987? Muitos culpam transações comerciais realizadas eletronicamente, mas os computadores já eram utilizados há muitos anos. Alguma razão específica para a queda da bolsa ter acontecido naquela data em especial?

- Por que as espécies ancestrais e ecossistemas permanecem estáveis em estado fóssil por milhões de anos?

De acordo com Waldrop (1992), num primeiro momento, as questões supracitadas têm a mesma resposta: 'ninguém sabe'. Algumas dessas questões nem mesmo parecem questões científicas. Entretanto, se analisadas criteriosamente, percebe-se que têm muito em comum: todas elas se referem a um sistema complexo, considerando-se que muitos dos agentes independentes interagem uns com os outros de diversas maneiras; em todos os exemplos, a riqueza das interações permite que o sistema como um todo passe por uma 'auto-organização espontânea'.

Inspirados nos estudos de Cameron (1999), Finch (2001, 2004), LarsenFreeman (1997, 2000, 2002a), Paiva (2002, 2005, 2006), Bowsfield (2004), Parreiras (2005) e Van Lier (1996, 2002), que utilizam a teoria da complexidade para iluminar seus estudos e reflexões sobre questões relacionadas ao ensino e à aprendizagem de línguas, propomos o seguinte desafio para a área da Lingüística Aplicada:

- por que é tão difícil prever resultados de aprendizagem em uma sala de aula presencial ou virtual onde os alunos aparentemente usufruem das mesmas condições: professor, instituição, nível social, materiais, recursos tecnológicos, abordagem pedagógica etc.?

\section{O pensamento científico: do clássico ao contemporâneo ${ }^{3}$}

Um dos primeiros movimentos científicos no Mundo Ocidental começou com a necessidade de explicar a natureza com base no menor número possível de elementos, representado pela escola de Pitágoras com

\footnotetext{
${ }^{3}$ As considerações sobre o pensamento científico aqui apresentadas foram elaboradas a partir dos estudos de MacGill (2005), Rossi (2001) e Prass (2005).
} 
promissores estudos como os de Euclides, na área de geometria, de Aristóteles, na gravidade, entre outros.

Tais iniciativas científicas tiveram um declínio na Idade Média, mas foram redescobertas com a conquista de novos mundos, dando origem ao Movimento Renascentista. René Descartes, um dos expoentes desse movimento no campo das ciências naturais, propõe pela primeira vez um método que estabelece regras formais de investigação científica, retomando o legado grego. A revolucionária proposta de Descartes conta com o apoio e adesão da comunidade científica da época, marcando o início da sistematização da pesquisa científica. Como exemplo de estudos que aderiram à metodologia cartesiana, podem-se citar os estudos sobre mecânica, de Galileu Galilei, os quais abrem novos horizontes para a matemática moderna e para a física experimental, servindo de fundamentação para estudos expressivos na física como, por exemplo, os estudos de Newton.

Foi com Newton, entretanto, que a ciência clássica chega ao seu apogeu. Newton, usando a intuição de Galileu de abstrair o que é relevante do mundo físico, o método de Descartes como guia metodológico de investigação, e a metodologia axiomática de Euclides para sistematizar suas descobertas, apresenta à comunidade científica os princípios matemáticos da filosofia natural.

O extraordinário sucesso dos trabalhos de Newton provocou uma profunda mudança na visão de mundo, o que Kuhn $(2005)^{4}$ chamaria de quebra de paradigma, com seus conceitos de espaço e tempo absoluto, que previam a existência do espaço, independente do observador e de um tempo que flui, homogeneamente, também desvinculado do observador. Esses princípios, associados à obra Princípios Matemáticos da Filosofia Natural, desenvolvida posteriormente por Newton, marcam o início de uma visão científico-filosófica de mundo, conhecida como determinismo mecanicista.

Um dos desdobramentos dessa visão é a tentativa de se descrever o todo reduzindo-o à investigação das partes isoladas, dando origem ao que se denomina reducionismo. A título de exemplo, basta pensarmos nas idéias newtonianas de que o Universo é um grande relógio ( $b i g$ clock), fazendo alusão ao fato de que para que possamos entender o funcionamento de um relógio, se faz suficiente estudar isoladamente suas partes constituintes.

\footnotetext{
${ }^{4}$ Thomas Kuhn, em seu texto seminal publicado inicialmente em 1962, foi o responsável por cunhar a expressão "quebra de paradigma".
} 
É a era quântica, todavia, que desponta no fim do século XIX e que conduz a física à sua fase contemporânea. As possíveis interpretações da mecânica quântica fazem florescer correntes científico-filosóficas que dão início às novas visões de mundo, gerando hipóteses que buscam a descrição da realidade objetiva.

Das correntes atuais encontradas na física contemporânea, pode-se mencionar a Ciência da Complexidade, que inova ao propor uma visão holística que incorpora a não-linearidade, a imprevisibilidade, o dinamismo da relação entre as partes, a alta sensibilidade às condições iniciais e autoorganização de um fenômeno.

\section{A nova ciência}

Os conceitos básicos que fundamentavam a concepção clássica do mundo encontraram, hoje, seus limites num processo teórico que os cientistas naturais não hesitam em chamar de metamorfose (PRIGOGINE; STENERS, 1984; PRIGOGINE, 1996). Essa concepção que prevê a redução de um conjunto de processos naturais a um pequeno número de leis foi abandonada. Não são mais as situações estáveis e permanentes que interessam, mas as evoluções, as crises e as instabilidades. Os pesquisadores das ciências naturais não estão mais interessados apenas no estudo do que permanece, mas também no estudo do que se transforma, das perturbações geológicas, climáticas, da evolução das espécies, da gênese, das mutações das normas que interferem nos comportamentos sociais.

Waldrop (1992) defende que a visão mecânica do universo foi considerada inadequada para descrever fenômenos complexos uma vez que o universo, ao mesmo tempo em que é integrado, é incompreensível em termos da mecânica e do pensamento linear, ${ }^{5}$ acolhendo, dessa forma, a inovação, a aprendizagem e a adaptação.

Waldrop afirma, ainda, que um sistema é complexo "no sentido em que muitos agentes independentes estão interagindo um com o outro em um grande número de formas" e, no mesmo parágrafo, ele inclui nos exemplos "os milhões de indivíduos mutuamente interdependentes que formam uma sociedade humana" (WALDROP, 1992, p. 11, tradução nossa). Assim, um sistema complexo é composto de elementos cuja interação

${ }^{5}$ Entende-se por pensamento linear as idéias que consideram causa e efeito diretamente proporcionais. 
produz um evento global que é diferente da soma de suas ações individuais (LEWIN, 1994; WALDROP, 1992).

Nos sistemas adaptativos complexos (SACs), não é possível realizar previsões de longo prazo, mas podem ser encontrados padrões que permitem, com certo grau de possibilidade de acerto, realizar previsões a curto prazo. A meteorologia, por exemplo, tendo como base padrões observados, realiza previsões para uma semana com uma margem de erro razoavelmente pequena. Previsões para meses ou anos são realizadas, mas a possibilidade de erro é bem maior, considerando que "estímulos pequenos podem levar a conseqüências dramáticas” (LEWIN, 1994, p. 23).

Além disso, esses tipos de sistemas possuem a capacidade de adaptação, o que leva à auto-organização e, conseqüentemente, à emergência de novos padrões e comportamentos (HOLLAND, 1997). Um SAC é composto de agentes que interagem dinamicamente e adaptam-se uns aos outros e ao ambiente, pois são sensíveis ao feedback e procuram acomodação mútua para obter a otimização dos benefícios que garantirão a sua sobrevivência. Essas interações e adaptações possibilitam que os agentes de um sistema se auto-organizem, criando novos padrões e comportamentos. Uma outra característica dos SACs é a capacidade dos agentes de antecipar eventos com base em experiências anteriores. Segundo Holland (1997, p. 25),

a infinidade de interações, modificações por alterações aprendidas, dá origem à capacidade única que canídeos, felinos, primatas e outros mamíferos possuem de anteciparem as conseqüências das suas ações através da modelação dos respectivos mundos.

Essa capacidade de antecipação, no entanto, não é uma especificidade dos mamíferos, mas uma característica geral dos SACs. Uma bactéria, por exemplo, "é capaz de se mover na direção de um gradiente químico, prevendo implicitamente que a comida se encontra nessa direção" (HOLLAND, 1997, p. 57).

A teoria da complexidade apóia-se na idéia de que a ordem emerge das interações entre os organismos ou agentes. Embora essas interações entre os agentes possam seguir regras simples, sistemas complexos são capazes de inovação por meio dos processos de adaptação e autoorganização que levam à emergência de novos padrões.

No contexto educacional, dentro dessa perspectiva, cabe questionar "como pode o pensamento complexo contribuir para a pesquisa educacional" e "como a pesquisa educacional contribui para o pensamento 
complexo" (DAVIS; SUMARA, 2006, p. 8, tradução nossa). As discussões propostas neste trabalho seguem a idéia defendida por Davis e Sumara de que "o pensamento complexo não surge sobre, mas surge entre outros discursos" e é impulsionado por atitudes de investigação que consideram que "as ações de alguns sistemas contribuem para a transformação de suas próprias possibilidades” (DAVIS; SUMARA, 2006, p. 8, tradução nossa).

\section{Estudos na Lingüística Aplicada sob a perspectiva da complexidade}

As teorias do caos e da complexidade têm sido foco de crescente interesse na área de Lingüística Aplicada (LA) e, mais especificamente, nos estudos sobre ensino e aprendizagem de línguas. A publicação do artigo de Larsen-Freeman (1997) 'Chaos/Complexity Science and Second Language Acquisition'na Applied Linguistics tem sido considerada como o marco da entrada dessas teorias no campo da LA. De fato, esse foi o primeiro trabalho especialmente devotado às teorias do caos e da complexidade como metáfora para a compreensão dos processos envolvidos na aprendizagem de línguas, publicado em um grande periódico especializado da área de LA.

No entanto, outros trabalhos anteriores já haviam refletido sobre alguns aspectos da teoria do caos como Bowers (1990), Diller (1990), Lewis (1993), Taylor (1993) e da complexidade por Syverson (1994), Connor-Linton (1995) e Van Lier (1996) e suas implicações para o ensino e para a aprendizagem de línguas.

O trabalho de Bowers, apresentado em 1990 na Georgetown University Round Table on Language and Linguistics, levanta algumas questões que a teoria do caos pode trazer para as pesquisas em ensino e aprendizagem de línguas. Bowers tem sido citado por outros autores, particularmente, no que se refere ao uso de metáforas advindas da teoria do caos para iluminar questões de interesse da Lingüística Aplicada. Esse autor ressalta a importância das metáforas, afirmando que "você não vê algo até que tenha as metáforas corretas para percebê-lo" (BOWERS, 1990, p. 132, tradução nossa). Ele afirma que as metáforas dominantes nas pesquisas em ensino e aprendizagem de línguas são essencialmente hierárquicas e lineares. Segundo ele as metáforas "ao mesmo tempo guiam e restringem a nossa maneira de pensar sobre ensino, aprendizagem, avaliação, língua, o professor, o aluno" (BOWERS, 1990, p. 128, tradução nossa). 
Diller (1990) e Lewis (1993), fundamentando-se na teoria do caos, incorporam o princípio da não-linearidade às suas discussões sobre o ensino e a aprendizagem de línguas. Diller discute a aprendizagem de línguas com foco nos métodos de ensino, enquanto Lewis concentra-se no desenvolvimento da sua abordagem lexical de ensino de línguas.

Taylor (1993) utiliza em sua tese a teoria do caos e a geometria fractal para analisar a escrita de alunos de inglês como segunda língua em conferências sincrônicas on-line. Esse autor ressalta que, em seu estudo, os métodos de análise não foram diretamente importados da teoria do caos. Essa teoria é utilizada essencialmente como uma metáfora para a análise dos textos eletrônicos. Segundo ele "apropriações responsáveis de teoria científica requerem que o pesquisador esclareça se a teoria está sendo usada metafórica ou metodologicamente" (TAYLOR, 1993, p. 148, tradução nossa).

Isso, no entanto, não desvaloriza, segundo o autor, a aplicação de metáforas advindas das ciências naturais a outras áreas. De fato, "as metáforas da ciência influenciam a construção de conhecimento na cultura mais ampla, e grandes metáforas culturais influenciam como os cientistas constroem suas pesquisas" (TAYLOR, 1993, p. 148-149, tradução nossa).

O autor conclui que o caos parece ter ocupado a posição de se tornar a metáfora dominante que tanto cria quanto gerencia a complexidade na cultura pós-moderna.

A tese de Syverson (1994) é, segundo Nelson (2002), a primeira aplicação empírica da teoria da complexidade nos estudos sobre o desenvolvimento da escrita. Sua análise pauta-se pelas práticas de escrita de um poeta, de acadêmicos e pesquisadores em uma lista de discussão via $e$-maile de estudantes de um curso de escrita em língua inglesa. Essa pesquisadora propõe o termo "ecologia da composição" como uma unidade diferente de análise que envolve a compreensão dos escritores, dos textos e da audiência e observa que o indivíduo e o ambiente se influenciam mutuamente em um processo interconectado de adaptação através do tempo.

Connor-Linton (1995) retoma e estende o modelo de Bowers, apoiando-se em Waldrop (1992) para abranger questões mais amplas da complexidade, como adaptação e auto-organização, não consideradas no trabalho de Bowers. Também percebe, após descobrir a literatura popular sobre a pesquisa em complexidade, que muitos aspectos descobertos pelos pesquisadores da ciência da complexidade proporcionavam um novo olhar 
sobre a linguagem e surpreendeu-se por essa noção, aparentemente, não estar sendo aplicada à linguagem (WALDROP, 1992). Porém, após a apresentação oral do seu trabalho, muitos lingüistas lhe informaram que trabalhavam com a noção de complexidade.

Após examinar as descrições e exemplos de complexidade oferecidos por Waldrop (1992) e acrescentar exemplos próprios, Connor-Linton discute as possíveis conexões da teoria da complexidade com a linguagem e o ensino de línguas. Segundo esse autor, seu objetivo não é construir uma nova teoria da "complexidade lingüística", mas "sugerir o valor potencial do construto da complexidade [...] para um número de questões em lingüística e em ensino de línguas" (CONNOR-LINTON, 1995, p. 596, tradução nossa).

Na mesma direção, Van Lier (1996), em sua discussão sobre a interação no currículo de ensino de línguas, compreende o contexto educacional - e a sala de aula - como um sistema complexo.

Esses trabalhos apontaram para algumas contribuições das teorias do caos e da complexidade para o ensino de línguas, contribuições essas que foram retomadas e ampliadas por Larsen-Freeman (1997). Embora tais trabalhos tenham tocado em alguns aspectos dessa questão, eles não causaram, na ocasião, grande repercussão na área. Assim, é pertinente dizer que foi Larsen-Freeman (1997) quem trouxe as teorias do caos e da complexidade para a arena da Lingüística Aplicada. Nesse artigo, a autora chama a atenção para diversas similaridades existentes entre os sistemas complexos encontrados na natureza e a aprendizagem de segunda língua.

Discutindo questões relativas à interlíngua, às diferenças individuais, aos efeitos da instrução, Larsen-Freeman defende que em sistemas não lineares, como a aprendizagem de segunda língua, o comportamento do todo emerge da interação das partes. Sendo assim, estudando as partes isoladamente, uma por uma, estaremos tratando de cada parte e não da maneira em que as partes interagem. A autora ainda nos chama a atenção para tendência de se buscarem soluções simples, prematuramente, para sistemas como a aprendizagem de segunda língua, que contam com problemas complexos.

Após a publicação do trabalho de Larsen-Freeman (2007), diversos trabalhos têm refletido sobre as implicações da complexidade para a compreensão das relações de ensino e aprendizagem de línguas. Uma das implicações dessa perspectiva, segundo a autora, é que desencoraja explicações reducionistas aos eventos de ensino e aprendizagem de línguas. 
Van Lier (1997) apresenta a perspectiva ecológica à aprendizagem de línguas como uma alternativa para analisar os contextos em que os usos e a aprendizagem de línguas estão situados. Sua abordagem ecológica da educação lingüística gira em torno de quatro perspectivas essenciais: a) a semiótica, ou a ciência da comunicação e usos de signos; b) a ecologia, ou o estudo dos inter-relacionamentos complexos entre os organismos no e com o seu ambiente; c) a interação e a ação significativas como investigadoras da aprendizagem; e d) as teorias do caos e da complexidade como modelos científicos para estudar os processos e desenvolvimentos que estão além dos mecanismos lineares causa-efeito ou input-output.

A abordagem ecológica da aprendizagem de línguas, desenvolvida sob a perspectiva sociocultural e apoiada na teoria da complexidade, propõe uma análise dos ambientes de aprendizagem que considere as diversas interações entre seus componentes e com o ambiente social mais amplo (VAN LIER, 1996, 1997, 2000, 2004; TUDOR, 2001, 2003; LAM; KRAMSCH, 2003). Nesse sentido, as interações dos estudantes com o processo de aprendizagem (inclusive com os demais participantes) surgem de um complexo de atitudes que são específicas a cada um como indivíduo, bem como de suas relações com o meio no qual estão inseridos (TUDOR, 2001). Nessa perspectiva, a sala de aula de línguas é compreendida como um sistema adaptativo complexo no qual interações em um nível local levam ao surgimento de propriedades emergentes em um nível global. Em um SAC, agentes individuais interagem entre si e adaptam-se uns aos outros e ao ambiente. Isso acontece porque os agentes são sensíveis ao feedbacke buscam a acomodação mútua e a otimização dos benefícios (NELSON, 2004). Essas interações e adaptações possibilitam que os agentes de um sistema se auto-organizem, levando, assim, à emergência de novos padrões e comportamentos.

Fundamentando-se no trabalho de Larsen-Freeman (1997) e diversos autores das teorias do caos e da complexidade, Paiva (2002) faz uma revisão dos principais modelos de aquisição de segunda língua e propõe um modelo baseado na teoria dos sistemas complexos. Este foi o primeiro trabalho realizado no Brasil na área de Lingüística Aplicada que procurou compreender o processo de desenvolvimento de uma segunda língua como um sistema complexo. O seu modelo, denominado Modelo Fractal de Aquisição de Linguas, foi desenvolvido em um trabalho posterior (PAIVA, 2005b) e incorporado em suas investigações sobre a questão da autonomia 
de aprendizes (PAIVA, 2006) e sobre o desenvolvimento das habilidades orais (PAIVA, no prelo). A partir de narrativas de aprendizagens de aprendizes de inglês, francês, alemão, italiano e espanhol, a pesquisadora encontra evidências de que a aprendizagem de uma segunda língua é um fenômeno complexo. Também observa que os processos de aprendizagem não são semelhantes, mas, sim, caóticos e imprevisíveis. O início de tudo, segundo ela, é a desordem, mas, dentro da aparente desordem, uma ordem se estabelece. A aprendizagem de línguas, como um fenômeno complexo, está em constante evolução e as alterações no sistema, que acontecem de forma imprevisível, podem promover mudanças que levarão a resultados inesperados.

Seguindo essa tendência, em 2002 é publicado o livro Language Acquisition and Language Socialization: ecological perspectives, que reuniu pesquisadores renomados em diversas áreas da LA, como psicolingüística, sociolingüística, lingüística antropológica e lingüística educacional. Embora com perspectivas diversas, os trabalhos reunidos nesse livro alinham-se à metáfora da "ecologia", procurando superar a corrente disputa entre os paradigmas da aprendizagem e da socialização. Tal metáfora, que "captura a interação dinâmica entre os usuários de uma língua e o ambiente como entre as partes de um organismo vivo, parece oferecer uma nova forma de reunir perspectivas de várias disciplinas para iluminar as complexas relações sob investigação" (KRAMSCH, 2002, p. 3).

O livro Ecology of Language Aqcuisition de Leather e Van Dam (2003) também agrupou pesquisadores com perspectivas diversas, porém sob a concepção de ecologia da aprendizagem de línguas e compreendendo os processos cognitivos dos indivíduos como intrinsecamente interligados com suas experiências no mundo físico e social. Reunindo trabalhos com perspectivas múltiplas, esse livro enfoca a emergência do desenvolvimento lingüístico por meio da interação dos aprendizes (crianças ou adultos) com o seu ambiente (espacial, social, educacional etc.) nos processos de aquisição de primeira língua, de segunda língua e, até mesmo, de aquisição de linguagem por parte de robôs.

Inspirados nas contribuições de Paiva (2002), Fleischer (2003) e Parreiras (2005) incorporaram as teorias do caos e da complexidade em suas investigações sobre eventos de ensino e aprendizagem, especialmente em contextos de educação on-line. Fleischer, em sua análise das contribuições da colaboração on-line para o desenvolvimento profissional 
de professores de línguas, analisa a aprendizagem a partir dos fundamentos da teoria do caos. Segundo ele, compreender a aprendizagem a partir dessa perspectiva "é útil na medida em (sic) que se deixa de buscar ou esperar resultados lineares e que se leva em consideração o grande número de fatores inter-relacionados ao se tentar deduzir relações de causa e efeito" (FLEISCHER, 2003, p. 18). Parreiras (2005) analisa a sala de aula on-line sob a perspectiva da teoria da complexidade e observa que os fluxos interacionais estabelecidos nas salas de aulas digitais se caracterizam como sistemas complexos, apresentando indícios de imprevisibilidade, sensibilidade às condições iniciais, interatividade, adaptabilidade e dinamismo e auto-organização.

Para Parreiras (2005), a sala de aula, na perspectiva dos sistemas complexos, funciona como organismo cujos elementos são responsáveis pela sua evolução, a qual se torna possível por meio das interações entre todos os fatores envolvidos. O autor ainda enfatiza que essa interação se dá tanto internamente (na sala de aula, por exemplo) quanto em sistemas externos (a própria escola, comunidade, sistema educacional) de forma dinâmica e interdependente.

O livro The Ecology and Semiotics of Language Learning: a sociocultural perspective de Van Lier (2004), oferece uma ampla e compreensiva abordagem da ecologia da aprendizagem, tanto em termos teóricos quanto em relação às suas implicações práticas. Incluindo a teoria sociocultural, a semiótica, a teoria da complexidade, entre outras, esse livro faz uma consistente revisão das teorias de aprendizagem de línguas e apresenta o desenvolvimento lingüístico (L1 e L2) como um fenômeno emergente. Essa perspectiva ecológica, segundo Van Lier, é "uma maneira de pensar sobre ensino e aprendizagem em toda a sua complexidade, uma forma de olhar para a linguagem como uma ferramenta de muitos usos e como uma componente chave em todas as atividades de construção de sentido humanas" (VAN LIER, 2004, p. 224, tradução nossa).

Em 2006, a revista Applied Linguistics dedicou uma edição especial integralmente às contribuições da Teoria da Complexidade para a Lingüística Aplicada, mais especificamente à compreensão da linguagem e da aprendizagem de línguas como fenômenos emergentes, partindo da premissa de que a linguagem é "um sistema dinâmico cujas propriedades em interação não são redutíveis à soma de suas partes" (COOK; KASPER, 2006, p. 554, tradução nossa). Os artigos dessa edição especial, informados 
por uma perspectiva emergentista, procuram "explicar as sistematicidades que surgem do desenvolvimento da língua do aprendiz de segunda língua, a criação e evolução de redes lexicais, a adaptação dinâmica que ocorre durante a interação e mesmo na evolução e mudança da língua" (ELLIS; LARSEN-FREEMAN, 2006, p. 578, tradução nossa). Essa visão emergentista da aprendizagem de línguas, segundo Cook e Kasper (2006), oferece uma solução coerente para diversas questões acerca do desenvolvimento lingüístico, tais como a divergência de estratégias desenvolvimentais, como o trabalho de Larsen-Freeman (2006), a multi-direcionalidade de transferência léxica, como o trabalho de Meara (2006), e o problema da pobreza ou indeterminação do estímulo levantado pela teoria gerativa, como o trabalho de Mellow (2006).

Nesse sentido, Ellis (2007) ressalta que, se acreditarmos que a sistematicidade e dinamicidade da aprendizagem de línguas não são guiadas por uma gramática universal nem se trata de simples imitação, precisaremos procurar explicações emergentistas.

Leffa (2006), apoiando-se nos trabalhos de Paiva (2002) e de LarsenFreeman (1997), parte do pressuposto de que a aprendizagem de línguas é um sistema muito complexo para que possa ser explicado por meio de uma única teoria de aprendizagem e propõe uma abordagem transdisciplinar na pesquisa e no ensino de línguas.

Motivados pela nova perspectiva e pelos estudos de Larsen-Freeman (1997, 2002a, 2006), Van Lier (1996, 1997, 2000, 2002, 2004), Tudor (2001, 2003), Fleischer (2003), Parreiras (2005), Paiva (2002, 2005a, 2006), entre

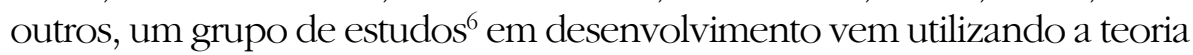
da complexidade como base epistemológica para a compreensão de fenômenos diversos relacionados aos processos de ensino e aprendizagem e, em particular, em contextos de educação on-line.

No contexto interacional mediado pela linguagem e tecnologia esse grupo conta com dois estudos empíricos voltados para a melhor compreensão do funcionamento de comunidades de aprendizagem: o primeiro, ${ }^{7}$ volta-se para investigações sobre a colaboração e desenvolvimento da reflexão crítica em grupos on-line que interagem sem a interferência

${ }^{6}$ Grupo de Pesquisa do Programa da Pós-Graduação em Estudos Lingüísticos da UFMG, orientado pela Prof. Dr. Vera Menezes de Oliveira e Paiva.

${ }^{7}$ Júnia de Carvalho Fidelis Braga, doutoranda do PosLin-UFMG. 
direta do professor; e o segundo ${ }^{8}$ busca compreender os eventos interativos ocorridos nas aulas on-line e face-a-face em um curso de escrita em língua inglesa como língua estrangeira. Em uma perspectiva ecológica, os participantes são compreendidos como essencialmente interligados entre si e com o seu ambiente. Assim, o ambiente de ensino e aprendizagem de línguas configura-se como um sistema complexo e suas atividades surgem e definem-se por processos de emergência, adaptação e auto-organização.

Ainda na perspectiva colaborativa on-line, um terceiro estudo? discute, à luz da teoria sócio-cultural e da perspectiva da complexidade, como o processo das trocas de e-mails provoca a dinâmica não-linear de um sistema (curso virtual) de interação sócio-educacional mediado por computador. Sua análise visa apontar não só as evidências que corroboram a natureza complexa da dinâmica não-linear (caótica) dos movimentos que configuram o sistema do curso mas também os aspectos que podem se configurar em categorias de andaimes - scaffolding - conceito tomado a partir das propostas de Wood, Bruner e Ross (1976) e de Cazden (1979), providos, colaborativamente, entre os participantes.

Dois outros estudos estão sendo desenvolvidos com foco em ensino e aprendizagem de línguas. Um deles ${ }^{10}$ apóia-se nas características dos Sistemas Complexos, descritas por Holland (1997), e nos conceitos de Atratores Estranhos e Fractais da Teoria do Caos, de acordo com Stewart (1991), e procura entender a inter-relação entre desenvolvimento e reconstrução de identidades pela perspectiva do Caos e dos Sistemas Complexos com foco na emergência de múltiplas identidades via discurso e demonstrando como a interação entre esses múltiplos "eus" pode tanto contribuir quanto impedir o processo de desenvolvimento de LE - entendido também como um sistema complexo. O outro estudo ${ }^{11}$ investiga o processo de evolução da competência comunicativa de professores de inglês inseridos

\footnotetext{
${ }^{8}$ Antônio Carlos Soares Martins, doutorando do PosLin-UFMG, afiliado à Universidade Estadual de Montes Claros e ao Centro Federal de Educação Tecnológica de Januária, MG.

${ }^{9}$ Valdir Silva, doutorando do PosLin-UFMG, afiliado à Universidade do Estado de Mato Grosso.

${ }^{10}$ Liliane Assis Sade, doutoranda do PosLin-UFMG, afiliada à Universidade Federal de São João Del Rei, MG.

${ }^{11}$ Rita de Cássia Augusto, doutoranda do PosLin-UFMG, afiliada ao Colégio Técnico da Universidade Federal de Minas Gerais.
} 
num contexto de educação continuada, fundamentando-se no modelo de competência comunicativa proposto por Bachman (1990) e nas teorias do caos e da complexidade. Sua pesquisa almeja refletir sobre alguns dados que evidenciam a não-linearidade do processo de desenvolvimento de uma língua estrangeira.

\section{Considerações finais}

As contribuições aqui discutidas parecem compartilhar o pensamento de que a perspectiva da complexidade nos encoraja a reconhecer a existência de relações que sustentam os fenômenos. Muito se pode aprender se dividirmos um determinado equipamento para vermos o que cada parte faz para que ele funcione, idéia original do reducionismo. Entretanto, nem tudo pode ser examinado dessa forma. Parafraseando MacGill (2005), dissecando um rato aprendemos sobre suas partes, mas dissecá-lo implica matá-lo, o que nos impede de saber o que lhe dá vida. Muitas vezes, se faz necessário tomar um posicionamento que contemple o dinamismo de um todo para que se possa melhor entender como suas partes funcionam e o que as interações dessas partes podem fazer emergir.

Trazido inicialmente para o campo da LA a partir de iniciativas isoladas, o paradigma da complexidade, gradativamente, tem se firmado como uma base epistemológica consistente para a compreensão dos contextos e eventos envolvidos nas atividades de ensino e aprendizagem de línguas. Esses eventos relativos ao processo de aprendizagem de segunda língua, assim como o universo, são essencialmente complexos.

A natureza complexa dos fenômenos levou os cientistas clássicos a estabelecer procedimentos metodológicos que reduziam os fenômenos à condição de análise vislumbrada então. Nas palavras de Kuhn, "essas restrições, nascidas da confiança no paradigma, revelaram-se essenciais para o desenvolvimento da ciência" (KUHN, 2005, p. 5).

Os avanços científicos e tecnológicos advindos desses esforços estabeleceram como paradigma confiável a visão reducionista da ciência clássica. A emergência de um outro olhar nos permite ver certos aspectos em um fenômeno, muitas vezes, não contemplados por um único paradigma. Essa idéia se alinha ao pensamento de Halliday (2001) de que o ensino de uma segunda língua é demasiadamente complexo e multifacetado para ser analisado a partir de uma única perspectiva, seja ela qual for. Nesse foco, Larsen-Freeman (2002b) ressalta que uma das 
contribuições do paradigma da complexidade é que ele possibilita não só rever nossas maneiras de conceitualização mas também nos permite perceber as concepções subjacentes aos paradigmas mais tradicionais.

Nessa direção, Lantolf (2002) nos lembra que o campo da LA está tão profundamente investido da metáfora computacional que concebe a mente como uma máquina, herdada da lingüística chomskiana e da ciência cognitiva, que ela é raramente reconhecida como metáfora. As metáforas, quando amplamente operacionalizadas nas culturas de pesquisa e na cultura em geral, acabam por se tornar invisíveis. Assim, corre-se o risco de as pessoas, não vendo suas próprias metáforas como tal, conceberem-nas como 'verdade'. Essas novas metáforas, portanto, podem possibilitar-nos perceber e questionar metáforas já naturalizadas em nossa tradição de pesquisa e, dessa forma, expandir a percepção da natureza complexa de nossos objetos de estudo.

Uma vez que o panorama apresentado envolve tanto questões teóricas quanto implicações pedagógicas, esperamos que ele possa servir de contribuição para novos desafios e questões para novos estudos na área da Lingüística Aplicada.

\section{Referências}

BACHMAN, L. Fundamental considerations in language testing. Oxford: Oxford University Press, 1990.

BATES, E.; ELMAN, J.; JOHNSON, M.; KARMILOFF-SMITH, A.; PARISI, D.; PLUNKETT, K. Innateness and emergentism. In: BECHTEL, W.; GRAHAM, G. (Ed.). A companion to cognitive science. Oxford: Blackwell, 1999. p. 590-601. BOWERS, R. Mountains are not cones: what can we learn from chaos? In: ALATIS, J. E. (Ed.). Linguistics, Language Teaching and Language Acquisition: The Interdependence of Theory, Practice and Research. Washington, D.C.: Georgetown University Press. 1990. p. 123-136. (Georgetown University Round Table on Language and Linguistics, 1990.). BOWSFIELD, S. Complexity in the English language arts classroom: prompting the collective. In: PROCEEDINGS COMPLEXITY SCIENCE AND EDUCATIONAL RESEARCH CONFERENCE, 2004, Chaffey's Locks. Canada, 2004. p. 147-154.

CAMERON, L. The complex dynamics of language use on tasks. 1999. Disponível em: <http://www.education.leeds.ac.uk/research/ljc_complang.pdf >. Acesso em: 25 nov. 2004. 
CAZDEN, C. Peekaboo as an instructional model: discourse development at home and at school. Stanford Papers and Reports in Child Language Development, v. 17, p. 1-19, 1979.

CONNOR-LINTON, J. Complexity, linguistics and language teaching. In: ALATIS, J. E.; STRAEHLE, C. A.; GALLENBERGER, B. (Ed.). Linguistics and the Education of Language Teachers: Ethnolinguistic, Psycholinguistic, and Sociolinguistic Aspects. Washington, D.C.: Georgetown University Press. 1995. p. 596-604. (Georgetown University Round Table on Language and Linguistics, 1995).

COOK, G.; KASPER, G. Editorial. Applied Linguistics, Oxford: Oxford University Press, v. 27, n. 4, p. 554-557, 2006. Special Issue.

DAVIS, B.; SUMARA, D. Complexity and education: inquiries into learning, teaching, and research. Mahwah, N.J.: Lawrence Erlbaum, 2006.

DEMO, P. Complexidade e aprendizagem: a dinâmica não linear do conhecimento. São Paulo: Atlas, 2002.

DILLER, K. The non-linearity of language-learning and "post-modern" language teaching methods. In: BURMEISTER, H.; ROUNDS, P. (Ed.). Variability in second language acquisition. Eugene Oregon University of Oregon, 1990. v. 2.

ELLIS, N. C. Emergentism, connectionism and language learning. Language Learning, Michigan: University of Michigan, v. 48, n. 4, p. 631-664, 1998. ELLIS, R. Second language acquisition. Oxford: Oxford University Press, 2003. ELLIS, N. C.; LARSEN FREEMAN, D. Language emergence: implications for applied linguistics. Introduction. Applied Linguistics, Oxford: Oxford University Press, v. 27, n. 4, p. 558-589, 2006. Special Issue.

ELLIS, N. C. Dynamic systems and SLA: the wood and the trees. Bilingualism: Language e Cognition, Cambridge: Cambridge University Press, v. 10, n. 1, p. 23-25, 2007.

FINCH, A. Complexity and systems theory: implications for the EFL teacher/ researcher. The Journal of Asia TEFL, n. 1/2, p. 27-46, 2004.

FINCH, A. Complexity in the language classroom. Secondary Education Research, v. 47, p. 105-40, 2001. Disponível em: <http://www.finchpark. com/arts/comp.htm>. Acesso em: 16 mar. 2005.

FLEISCHER, E. A colaboração on-line como subsídio para o desenvolvimento profissional de professores de linguas. 2003. Dissertação (Mestrado) Faculdade de Letras da UFMG, Belo Horizonte, 2003. 
GLEICK, James. Caos: a criação de uma nova ciência. 14. ed. Rio de Janeiro: Campos, 1989. Original inglês.

HALLIDAY, M. A. K. New ways of meaning: the challenge to applied linguistics. In: FILL, A.; MÜHLHÄUSLER, P. (Ed.). The ecolinguistics reader. language, ecology and environment. London: Continuum, 2001, p. 175-202. HOLLAND, J. H. A ordem oculta: como a adaptação gera a complexidade. Trad. de José Luís Malaquias. Lisboa: Gradiva, 1997.

HOLLAND, J. H. Emergence: from chaos to order. Oxford: Oxford University Press, 1998.

HOLLAND, J. H. Studying complex adaptive systems. Journal of Systems Science and Complexity, Boston: Srpinger, v. 19, p. 1-8, 2006.

KRAMSCH, C. (Ed.). Language acquisition and language socialization: ecological perspectives. London: Continuum, 2002.

KUHN, T. S. A estrutura das revoluções científicas. 9. ed. São Paulo: Perspectiva, 2005.

LAM, E.; KRAMSCH, C. The ecology of an SLA community in a computermediated environment. In: LEATHER, J; VAN DAM, J. (Ed.). Ecology of language acquisition. Amsterdam: Kluwer Academic, 2003.

LANTOLF, J. Part One: commentaries. In: KRAMSCH, C. (Ed.). Language acquisition and language socialization: ecological perspectives. London: Continuum, 2002. p. 88-95.

LARSEN-FREEMAN, D. Chaos/complexity science and second language acquisition. Applied Linguistics. Oxford: Oxford University Press, v. 2, n. 18, p.141-165, 1997.

LARSEN-FREEMAN, D. Second Language Acquisition and Applied Linguistics. Annual Review of Applied Linguistics, Cambridge: Cambridge University Press, v. 20, p. 165-181, 2000.

LARSEN-FREEMAN, D. Language acquisition and language use from a chaos/complexity theory perspective. In: KRAMSCH, C. (Ed.). Language acquisition and language socialization. London: Continuum, 2002a. p. 33-46. LARSEN-FREEMAN, D. Part One: commentaries. In: KRAMSCH, C. (Ed.). Language acquisition and language socialization: ecological perspectives. London: Continuum, 2002b. p. 88-95.

LARSEN-FREEMAN, D. The emergence of complexity, fluency, and accuracy in the oral and written production of five chinese learners of English. Applied Linguistics, Oxford: Oxford University Press, v. 27, n. 4, p. 590-619, 2006. 
LEATHER, J; VAN DAM, J. (Ed.). Ecology of language acquisition. Amsterdam: Kluwer Academic, 2003.

LEFFA, V. J. Transdisciplinaridade no ensino de línguas: a perspectiva das teorias da complexidade. Revista Brasileira de Lingüistica Aplicada, Belo Horizonte: Universidade Federal de Minas Gerais, v. 6, n. 1, p. 27-49, 2006.

LEWIN, Roger. Complexidade: a vida no limite do caos. Trad. Marta Rodolfo Schimidt. Rio de janeiro: Rocco, 1994.

LEWIS, M. The lexical approach: the state of ELT and the way forward. Hove: Language Teaching Publ., 1993.

LORENZ, E. N. The essence of chaos. Seattle: Univ. Washington Press, 1993.

MACGILL, V. A history of chaos and complexity. Disponível em: <http:// complexity.orcon.net.nz/history.html>. Acesso em: 20 maio 2005.

MALLOWS, D. Non-linearity and the observed lesson. ELTJournal, Oxford: Oxford University Press, v. 56, n. 1, p. 3-10, 2002.

MEARA, P. Emergent properties of multilingual lexicons. Applied Linguistics, Oxford: Oxford University Press, v. 27, n. 4, p. 620-644, 2006. MELLOW, J. D. The emergence of second language syntax: a case study of the acquisition of relative clauses. Applied Linguistics, Oxford: Oxford University Press, v. 27, n. 4, p. 645-670, 2006.

MORIN, E. Introdução ao pensamento complexo. Lisboa: Instituto Piaget, 1990.

MORIN, E. Ciência com consciência. Tradução de Maria D. Alexandre e Maria Alice Dória. Rio de Janeiro: Bertrand Brasil, 1998.

NELSON, C. P. Contradictions in learning to write in a second language classroom: insights from radical constructivism, activity theory, and complexity theory. 2002. Tese (Doutorado) - University of Texas at Austin, Austin, 2002.

NELSON, C. P. The role of networks in learning to write. In: COMPLEXITY SCIENCE AND EDUCATIONAL RESEARCH CONFERENCE, 2004, Chaffey's Locks, Proceedings... Canada. p. 91-105.

NELSON, C. P. Building blocks and learning. Cumplicity: an International Journal of Complexity and Education, Edmont, Alberta: University of Alberta, v. 1, n. 1, p. 39-55, 2005.

PAIVA, V. L. M. de O. Caleidoscópio: fractais de uma oficina de ensino aprendizagem: memorial. Belo Horizonte: Faculdade de Letras, Universidade Federal de Minas Gerais, 2002. 
PAIVA, V. L. M. de O. Autonomia e complexidade: uma análise de narrativas de aprendizagem. In: FREIRE, M. M; ABRAHÃO, M. H. V; BARCELOS, A. M. F (Org.). Lingüistica aplicada e contemporaneidade. Campinas; São Paulo: Pontes: ALAB, 2005a. p. 135-153.

PAIVA, V. L. M. de O. Modelo fractal de aquisição de línguas. In: BRUNO, F. C. (Org.). Ensino-aprendizagem de linguas estrangeiras: reflexão e prática. São Carlos: Claraluz, 2005b, p. 23-36.

PAIVA, V. L. M. de O. Autonomia e complexidade. Linguagem e Ensino, Pelotas: Universidade Católica de Pelotas,v. 9, n.1, 77-127, 2006.

PAIVA, V. L. M. de O. As habilidades orais nas narrativas de aprendizagem. Trabalhos em Lingüística Aplicada. No prelo.

PARREIRAS, V. A. A sala de aula digital sob a perspectiva dos sistemas complexos: uma abordagem qualitativa. Tese (Doutorado) - Faculdade de Letras da UFMG, Belo Horizonte, 2005.

PRASS, A. História da física. Disponível em: <http://fisicanet.terra.com.br/ / historia>. Acesso em: 10 jun. 2005.

PRIGOGINE, I.; STENERS, I. A nova aliança: a metamorfose da ciência. Brasília: Ed. Universidade de Brasília, 1984.

PRIGOGINE, I. Ofim das certezas: tempo, caos e as leis da natureza. São Paulo: Ed. Unesp, 1996.

ROSSI, P. O nascimento da ciência moderna na Europa. Tradução de Antonio Angonese.Bauru: EDUSC, 2001.

STEWART, I. Será que Deus joga dados? A matemática do caos. Rio de Janeiro: Jorge Zahar, 1991.

SYVERSON, M. A. A wealth of reality: an ecology of composition. 1994. Tese (Doutorado) - UMI: University of California at San Diego, San Diego, 1994.

TAYLOR, P. H. Computer conferencing and chaos: a study in fractal discourse. 1993. Tese (Doutorado) - University of Texas at Austin, Austin, 1993.

TUDOR, I. The dynamics of the language classroom. Cambridge: Cambridge University Press, 2001.

TUDOR, I. Learning to live with complexity: towards language teaching. System, v. 31, p. 1-12, 2003.

VAN LIER, L. The classroom and the language learner. ethnography and second language acquisition. London: Longman, 1988.

VAN LIER, L. Interaction in the language curriculum: awareness, autonomy and authenticity. London; New York: Longman, 1996. 
VAN LIER, L. Approaches to observation in classroom research: observation from an ecological perspective. TESOL Quarterly, New York Teachers of English to Speakers of Other Languages, Inc., v. 31, p. 783-787, 1997.

VAN LIER, L. From input to affordance: social-interactive learning from an ecological perspective. In: LANTOLF, J. P. (Ed.). Sociocultural theory and second language learning. Oxford: Oxford University Press, 2000. p. 245-259. VAN LIER, L. An ecological-semiotic perspective on language and linguistics. KRAMSCH, C. (Ed.). Language acquisition and language socialization: ecological perspectives. London: Continuum, 2002. p. 140-164. VAN LIER, L. The ecology and semiotics of language learning: a sociocultural perspective. Boston: Kluwer Academic, 2004.

WALDROP, M. Complexity: the emerging science at the edge of order and chaos. New York: Simon e Schuster, 1992.

WOOD, D.; BRUNER, J.; ROSS, G. The role of tutoring in problem solving. Journal of Child Psychology and Psychiatry, Cambridge: Cambridge University Press, v. 17, p. 89-100, 1976. 